\title{
Intact Ability to Learn Internal Models of Arm Dynamics in Huntington's Disease But Not Cerebellar Degeneration
}

\author{
Maurice A. Smith and Reza Shadmehr \\ Laboratory for Computational Motor Control, Department of Biomedical Engineering, Johns Hopkins School of Medicine, \\ Baltimore, Maryland
}

Submitted 10 September 2004; accepted in final form 23 December 2004

Smith, Maurice A. and Reza Shadmehr. Intact ability to learn internal models of arm dynamics in Huntington's disease but not cerebellar degeneration. J Neurophysiol 93: 2809-2821, 2005. First published December 29, 2004; doi:10.1152/jn.00943.2004. Two different compensatory mechanisms are engaged when the nervous system senses errors during a reaching movement. First, on-line feedback control mechanisms produce in-flight corrections to reduce errors in the on-going movement. Second, these errors modify the internal model with which the motor plan is transformed into motor commands for the subsequent movements. What are the neural mechanisms of these compensatory systems? In a previous study, we reported that while on-line error correction was disturbed in patients with Huntington's disease (HD), it was largely intact in patients with cerebellar degeneration. Here we altered dynamics of reaching and studied the effect of error in one trial on the motor commands that initiated the subsequent trial. We observed that in patients with cerebellar degeneration, motor commands changed from trial-to-trial by an amount that was comparable to control subjects. However, these changes were random and were uninformed by the error in the preceding trial. In contrast, the change in motor commands of HD patients was strongly related to the error in the preceding trial. This error-dependent change had a sensitivity that was comparable to healthy controls. As a result, HD patients exhibited no significant deficits in adapting to novel arm dynamics, whereas cerebellar subjects were profoundly impaired. These results demonstrate a double dissociation between on-line and trial-to-trial error correction suggesting that these compensatory mechanisms have distinct neural bases that can be differentially affected by disease.

\section{IN T R O D U C T I O N}

Theoretical studies of motor control have proposed that the brain generates motor commands as a consequence of computations that resemble control policies and internal models (Shadmehr and Wise 2005). Control policies allow the brain to select goals and plan actions, while internal models compute motor commands that are appropriate for those plans and monitor sensory feedback so to update the plans. For example, when the goal is to reach a target, the motor system may evaluate the current state of the limb with respect to the goal and use a control policy to plan a small change in hand position (Hoff and Arbib 1993; Todorov and Jordan 2002). It may use an internal model of limb's inverse dynamics (called an inverse model) to convert that plan into motor commands (Atkeson 1989; Kawato 1989; Shadmehr and Mussa-Ivaldi 1994). It may use an internal model of limb's forward dynamics (called a forward model) to predict the sensory consequences of the

Address for reprint requests and other correspondence: M. Smith, Johns Hopkins School of Medicine, 416 Traylor Bldg., 720 Rutland Ave., Baltimore, MD 21205 (E-mail: msmith@bme.jhu.edu). motor commands (Jordan and Rumelhart 1992; Wolpert et al. 1995) and compare this prediction with sensory feedback (Miall and Wolpert 1996) to re-estimate current hand position with respect to the goal and update the motor plan (Bhushan and Shadmehr 1999) by issuing an error-dependent response aimed at correcting the ongoing movement.

We have previously showed that error feedback control is disturbed throughout the course of Huntington's disease-as much as 7-11 yr before onset of clinical symptoms. But surprisingly little is known about the neural mechanisms of supraspinal real-time feedback control during voluntary movement. How does the brain perform this real-time error feedback control? Ideas from computational motor control provide a framework for considering this problem. The simplest control method would be to generate corrective responses proportional to sensed error. However, significant delays that exist in the sensorimotor loop would require the gain of these responses to be quite small to maintain stability of the arm. These delays may be effectively compensated if the motion state of the arm can be predicted at the time point when compensatory motor commands would take effect. Such a predictor has been termed a forward model of dynamics (Jordan and Rumelhart 1992; Wolpert et al. 1995), and it can be computationally implemented using delayed sensory feedback, knowledge of the recent history of motor output, and knowledge of how the arm is likely to respond to this motor output (Bhushan and Shadmehr 1999). A good forward model can reduce delay driven instability allowing high feedback gains and powerful corrective responses based on simple linear responses to error, but these responses may not maximize the smoothness or efficiency of movement.

Recent theoretical work has suggested that the same computational mechanism that initially plans movement can be used to provide optimally efficient corrections to ongoing movements that experience error. The idea is that motion trajectories or motor commands themselves can be constantly recomputed so as to maximize the efficiency of the remaining portion of a movement at any time during its course. Alternate versions of a neural mechanism that could provide this computation have been termed control policies (Ijspeert et al. 2002; Shadmehr and Wise 2004), next state planners (Hoff and Arbib 1993), or optimal feedback controllers (Todorov and Jordan 2002). Although definitive evidence of this type of real-time re-planning has yet to be provided, this work suggests the

The costs of publication of this article were defrayed in part by the payment of page charges. The article must therefore be hereby marked "advertisement" in accordance with 18 U.S.C. Section 1734 solely to indicate this fact. 
possibility of a strong link between trajectory planning and error feedback control.

While such theories of computational motor control have been primarily applied to behavioral studies in healthy individuals, recent work has begun to apply the theories to patient studies (Desmurget et al. 2004; Smith et al. 2000; Wolpert et al. 1998). The aim is to use the theories to help identify computations that are affected when there is damage to specific parts of the motor system.

Studies of reaching in cerebellar patients are a good example of this approach. These patients exhibit errors in their reaching. The error patterns suggest that their motor commands do not predicatively compensate for interaction torques inherent in multi-joint motion (Bastian et al. 1996, 2000; Goodkin et al. 1993; Topka et al. 1998), suggesting that reaching deficits in cerebellar patients may be due in part to a malfunctioning inverse model.

Behavioral studies suggest that when a force field is imposed on the hand, the inverse model adapts (Conditt et al. 1997; Shadmehr and Mussa-Ivaldi 1994). Kawato and Gomi (1992) were first to propose that the cerebellum may play a dominant role in representing the inverse model. Using functional imaging, Nezafat et al. (2001) found activation of the ipsilateral cerebellum during force-field learning. This suggested that cerebellar patients might have difficulty adapting their motor commands when limb dynamics are altered. A recent study of patients with cerebellar degeneration confirmed that prediction (Maschke et al. 2004).

How specific is this inability to adapt to altered limb dynamics to cerebellar patients? Here we examined both a group of patients with cerebellar degeneration and a group of patients with Huntington's disease (HD). Imaging studies suggest that HD begins as primarily a basal ganglia disorder that affects the putamen (Aylward et al. 1994, 1998; Harris et al. 1996). Early in the disease course, rapidly alternating movements are slowed or disrupted (Hefter et al. 1987; Kirkwood et al. 2000 Penney et al. 1990). Patients become somewhat clumsy and may have trouble with fine motor tasks such as tying shoelaces, buttoning clothing, or performing needlework (Thompson 1988). We recently examined reaching movements in these patients and observed a disturbance in error feedback control: errors in the early part of a movement were poorly compensated by the motor commands in the remainder of the movement (Smith et al. 2000). This suggested that one of the many computational mechanisms that are involved in error feedback control was affected by damage to the basal ganglia.

However, error in a given movement not only requires a motor response during the same movement, it also requires a response in the subsequent movement: the error changes the inverse model that is thought to be used by the brain to compute the motor commands that initiate the subsequent movement (Thoroughman and Shadmehr 2000). Therefore here we asked whether the abnormal on-line response to error in HD was extended to include trial-to-trial mechanisms of adapting inverse models. We studied a group of HD and cerebellar patients that were roughly comparable in their movement disorder during reaching. Despite this impairment, HD patients adapted normally to altered limb dynamics while cerebellar patients were profoundly impaired.

\section{METHODS}

\section{Subjects}

Eight individuals positive for the HD genetic mutation and symptomatic with HD (median age: $51 \mathrm{yr}$ ), 15 asymptomatic gene carriers (pre-HD, median age: 34 ), 5 subjects with cerebellar lesions (median age: 41 ), and 14 control subjects (median age: 37 ) participated in the experiment. All subjects used their dominant hand, and all subjects but one asymptomatic gene carrier were right handed. The protocols were approved by the Johns Hopkins Institutional Review Board.

Patients suffering from HD have alterations in the IT-15 gene (Gusella et al. 1983), located on the short arm of chromosome 4. Close to the beginning of this gene is a region that includes a variable number of trinucleotide (the cytosine/adenine/guanine, CAG) repeats. The CAG trinucleotide encodes the amino acid glutamine. The difference between the IT-15 gene in unaffected and affected individuals is the length of this trinucleotide repeat. A genetic test to determine the repeat length was conducted at the Johns Hopkins Huntington's Disease Project. Subjects with a CAG repeat length of $\geq 37$ were called mutation-positive. The genetic test was part of the HD Presymptomatic Testing Program headed by Jason Brandt at Johns Hopkins School of Medicine. In this program, mutation-positive individuals participated in a longitudinal study that included annual psychiatric and neurological evaluations, along with a brain MRI. AGCs that participated in our study were part of this program.

Clinical evaluation of the pre-HD and HD subjects included a quantitative neurological examination (QNE) (Folstein et al. 1983). QNE includes an assessment of voluntary motor function via a motor impairment score (MIS) and an assessment of involuntary motor function via a chorea scale. Preliminary results from 17 AGC subjects who were studied annually at the Johns Hopkins Huntington's Disease Project show that individuals who are five or more years from predicted onset of the disease have QNE scores of $\leq 6$. This score gradually increases so that by the year of the onset QNE is $\sim 15$. In the early years of the disease development when the motor symptoms are mild, QNE is $<30$. All but one of the presymptomatic subjects we studied had QNE scores of $\leq 10$ (mean $=5.9 \pm 3.5$, mean $\pm \mathrm{SD}$; median $=6$ ). Symptomatic HD individuals that we studied were classified as mild to moderately affected (mean QNE $=29.1 \pm 14.7$, median $=27$ ).

All five cerebellar subjects were clinically diagnosed with cerebellar degeneration, were classified by their neurologist as having "pure cerebellar symptoms" and had atrophy limited to the cerebellum on MRI. Brief descriptions of MRI findings and dates of symptom onset are provided for these patients in the following text. KH: MRI documented cerebellar atrophy (SCA3) especially in the hemispheres, tested 9 years after initial symptoms. RR: MRI documented bilateral atrophy of the cerebellar hemispheres, tested seven years after initial symptoms. PC: cerebellar degeneration of undermined etiology confirmed by MRI to be localized specially along the vermis, tested 9 years after initial symptoms. CM: MRI confirmed idiopathic cerebellar degeneration with moderate bilateral atrophy of cerebellar hemispheres tested $5 \mathrm{yr}$ after initial symptoms. DB: MRI confirmed idiopathic cerebellar degeneration particularly along the vermis tested 5 yr after initial symptoms.

\section{Task}

We studied the standard force field adaptation paradigm $\{$ Shadmehr and Mussa-Ivaldi 1994\}. Briefly, subjects held the handle of a manipulandum. A small cursor $(4 \mathrm{~mm})$ indicated the subject's hand position and was displayed on a vertically oriented computer monitor in front of the subject (refresh rate of $60 \mathrm{~Hz}$ ). They reached to $1 \mathrm{~cm}^{2}$ targets that were spaced $10 \mathrm{~cm}$ apart from each other. The manipulandum measured hand position and velocity (sampled at $100 \mathrm{~Hz}$, accuracy: $>0.1 \mathrm{~mm}$ and $1.3 \mathrm{~mm} / \mathrm{s}$ ) and applied forces to the hand. 
Forces on the hand were perpendicular to hand velocity, producing a curl field. The relationship between force $(\mathbf{F})$ and velocity $(\mathbf{V})$ vectors was determined by matrix $C_{A}=\left[\begin{array}{llll}0 & 13 ;-13 & 0\end{array}\right] \mathrm{N} . \mathrm{s} / \mathrm{m}$ via the relationship $\mathbf{F}=C_{A} * \mathbf{V}$. We considered two kinds of fields: a clockwise curl field $C_{A}$ and a counter-clockwise curl field $C_{B}=-C_{A}$. We refer to these force fields as field $\mathrm{A}$ and field $\mathrm{B}$, respectively.

The experiment was divided into short sets of 96 or 100 trials, each a reach to a target. Sets generally took $6-8 \mathrm{~min}$ to complete. The first four sets were performed in the null field with the robot motors disabled. The next three to four sets were performed in field A, followed by one to four sets in field B. During the field sets, catch trials were inserted at a probability of $1 / 6$. In a catch trial, the field was turned off.

We instructed subjects to "make a quick movement to the target." We instructed them that the reaction time was not important- they could wait as long as they wished after target appearance before starting each movement-but when ready, they were to move in a rapid motion toward each target. The peak speed of each movement was displayed to the experimenter after each trial, and subject was coached to move faster or slower if movement speeds were outside the range of $0.25-0.55 \mathrm{~m} / \mathrm{s}$. The endpoint of each movement was used as the starting point for the subsequent movement, and movements were made in eight target directions.

\section{Analysis of field blocks}

We focused on motor commands that initiated a movement and defined aiming error $y$ as the angle between the net motion direction during the first $300 \mathrm{~ms}$ of movement and the target direction with the convention that counterclockwise errors are positive and movement onset defined as the first time that the speed of hand motion exceeded a threshold of $0.03 \mathrm{~m} / \mathrm{s}$ for $200 \mathrm{~ms}$ consecutively. There are at least two different ways in which the CNS may reduce these errors. When forces are applied to the hand in field trials, stiffening of the limb will produce reduction in reaching errors. However, this strategy will not result in errors during catch trials. Alternately, learning of an internal model of the forces will also result in a reduction in errors during field trials. However, this strategy will produce large errors in catch trials. Learning an internal model that predictively compensates for an external force-field will produce the same motor output on field and catch trials because the catch trials cannot be predicted. For this reason, early movement errors (before feedback compensation) will be shifted in the same direction for both force-field trials and catch trials. Although, this will reduce the magnitude of errors on force-field trials and will increase the magnitude of errors on catch trials, the difference between these errors may not change. The difference between error on force-field trials and catch trials corresponds to the effect of the force-field on error. In our experiment the size of this effect depends on the magnitude of the force-field and the net compliance of the arm. Although the force-field magnitude is held constant across subjects, arm compliance may vary across subjects, resulting in subject-dependent force-field effects. For this reason, it is useful to create a measure of learning that is independent of force-field effect size. Because catch trial errors indicate internal model formation but their magnitude depends on the size of the force-field effect, we defined a learning index that normalizes catch trial error size by an estimate of the force-field effect

$$
\text { Learning index }=\frac{y_{\mathrm{c}}}{\left|y_{\mathrm{c}}-y_{\mathrm{f}}\right|}
$$

This measure is similar to that used by Criscimagna-Hemminger et al. (2003). Here, $y_{\mathrm{c}}$ and $y_{\mathrm{f}}$ are errors in catch and field trials, respectively. Both of these measures of error were adjusted for any bias that may have been present during the last null set. Therefore errors in a field set always refer to change from errors in the null set. Note that if the force field is fully compensated, errors on field trials, $y_{\mathrm{f}}$, will be zero resulting in a learning index of magnitude 1 , whereas when no force-field adaptation occurs errors on catch trials, $y_{\mathrm{c}}$, will be zero resulting in a learning index of 0 . Note that if motor commands, on average, compensate for $50 \%$ of the forces in field $\mathrm{A}$, then and the learning index magnitude is 0.5 . Because of our sign conventions for error, in field A the learning index approaches 1 as errors in field trials decrease and errors in catch trials increase, whereas in field $\mathrm{B}$, this index approaches -1 .

We also measured movement duration and relative path length of each movement. Movement duration is quantified as the time elapsed between movement onset and termination. We defined onset as the first time that the velocity magnitude exceeded a threshold of $0.03 \mathrm{~m} / \mathrm{s}$ and remained above it for $200 \mathrm{~ms}$ consecutively, and we defined movement termination as the first time after onset that the movement speed went below the $0.03-\mathrm{m} / \mathrm{s}$ threshold and remained there for 200 ms consecutively. Relative path length was the distance traveled along each movement path divided by the straight-line distance between the start and endpoint of motion.

\section{Analysis of null blocks}

We observed that all subjects exhibited a similar pattern of errors when training began in the null set. We suspected that this was due to the unfamiliarity of the subjects to the passive dynamics of the robot. That is, in the null set, the motor system needed to adapt to the small but nontrivial inertial dynamics of the robot arm. To test for this, we simulated dynamics of a human arm holding onto our robot handle while reaching movements were performed to targets. Because the human arm was constrained to move in the horizontal plane during our task, the human and robot arms were modeled as coupled coplanar two link systems. The structure of the model and the parameters were described earlier (Shadmehr and Brashers-Krug 1997). Briefly, this model was parameterized by the inertia and physical dimensions of the robot and human arm, and by the stiffness and viscosity of the human arm. We used 34 and $35 \mathrm{~cm}$ for the human upper arm and forearm lengths, respectively, and used the following matrices to represent human joint-based arm stiffness and viscosity, respectively (expressed in absolute joint coordinates)

$$
\begin{aligned}
& \text { stiffness }=\left[\begin{array}{cc}
25 & -14 \\
-14 & 19
\end{array}\right] \mathrm{N} / \mathrm{m} \\
& \text { viscosity }=\text { stiffness } * 0.15 \mathrm{~s}
\end{aligned}
$$

Movement simulations were performed using MATLAB Simulink with a 2-ms sampling interval. We first computed the feed-forward human elbow and shoulder torques required to make perfect straightline minimum-jerk hand motion with a peak velocity of $0.375 \mathrm{~m} / \mathrm{s}$ to each target location in the condition that the human arm was not holding the robot arm. We then applied these joint torques to our model (with the human arm holding the robot arm) and computed the aiming errors in the resulting imperfect hand trajectories.

\section{Trial-by-trial effect of error}

Adaptation is partly due to the influence of errors in one trial on the motor commands that initiate the subsequent trial. To quantify adaptation nonparametrically, we compared all movement triplets in each target direction that were force-catch-force (FCF) or force-force-force (FFF). Using these force-any-force (FXF) triplets, we looked at the relationship between error in the middle movement $y^{(n+1)}$ and the change in error from the first movement $y^{(n)}$ to the third movement $y^{(n+2)}$. The change in performance, i.e., $y^{(n+2)}-y^{(n)}$, might have been due to random noise or due to the errors experienced on trials $n+1$, i.e., $y^{(n+1)}$. In an adaptive system, a positive $y^{(n+1)}$ generally results in a negative $y^{(n+2)}-y^{(n)}$ and the greater this sensitivity, the faster the learning. To measure the sensitivity, we computed the slope of the linear fit to the relationship between 
$y^{(n+2)}-y^{(n)}$ and $y^{(n+1)}$ in each subject. The distribution of sensitivities for each group was then compared across groups. Note that the triplets consisted of consecutive trials in the same direction, and so movements in other directions occurred between the elements of each triplet. However, we expected that errors experienced in directions other than the triplet direction would have smaller effects and these errors would at steady state be independent of the errors in the triplet direction and thus should not substantially bias our estimates of within-direction error sensitivity.

We used an ANOVA to compare the effect of group on the measured variables and post hoc $t$-test to compare pairs of groups. All $P$ values resulting from multiple comparisons were corrected using the Holm method (Holm 1979) to sequentially adjust the $P$ values deemed significant $(P<0.05)$.

\section{State-space model of trial-to-trial error}

The triplet analysis described in the preceding text assessed the trial-by-trial effect of error by relating performance changes in a certain movement direction to errors experienced in that same direction. However, errors made in other movement directions may also influence (albeit to a lesser degree) these performance changes. A recent theory describes how one can account for the effect of error experienced in any movement on any other movement (Donchin et al. 2003; Thoroughman and Shadmehr 2000). The idea is to fit a simple linear state-space model of direction-dependent error-driven learning to the full trial-to-trial sequence of aiming errors displayed by each subject during the force-field sets

$$
\begin{aligned}
\hat{y}(n) & =D_{\mathrm{d}} \cdot f(n)-z_{\mathrm{d}}(n) \\
Z_{\mathrm{d}}(n+1) & =Z_{\mathrm{d}}(n)+B \cdot \hat{y}(n)
\end{aligned}
$$

where $\hat{y}(n)$ is the model's estimate of error on the $n$th trial. The movement at the $n$th trial was toward direction $\mathrm{d}, f(n)$ is the force field magnitude on the $n$th trial, $D_{\mathrm{d}}$ is the arm compliance in direction d, and $z_{\mathrm{d}}(n)$ is the (learned) motor output that opposes the force-field on trial $n$. This model has two parts. The first part predicts error on a particular trial based on the force field applied and the estimated motor output on that trial. Here the predicted error is the difference between the effect of the force-field, $D_{\mathrm{d}} \cdot f(n)$, and the compensatory motor output on that trial. The second part of the model predicts motor output state on the next trial based on the state of motor output in the current trial, the error experienced in the current trial, and the error generalization parameter $B$. This second equation predicts that the change in motor output state on a particular trial is the product of the error generalization parameter and the error experienced on that trial. Note that the first equation in the model is scalar, whereas the second is an eight-dimensional vector equation (because there are 8 movement directions). In this equation, motor output state is updated for all possible directions of movement on every trial, but only the motor output state corresponding to a particular trial's movement direction contributes to the predicted error for that movement.

This type of autoregressive computational modeling of the trial-totrial changes in motor output has been previously used to characterize properties of error-dependent motor adaptation in healthy human subjects (Donchin et al. 2003; Scheidt et al. 2001; Thoroughman and Shadmehr 2000) Our model is most similar to the model of Thoroughman and Shadmehr (2000) but has a few key differences: $D_{\mathrm{d}}$ is now constrained to correspond to the pattern of effective compliance conferred by a two-dimensional compliance matrix (see derivation in the following text), the trial-to-trial sequence of errors corresponds to angular aiming errors rather that perpendicular displacements, and the current model was fit to data from individual subjects rather than to a data set averaged across subjects. This was necessary because we could not assume that the patient groups were homogenous. Note that fitting such models to across-subject averaged data will not produce the same results as the average of the fits to individual subject data because state-space model fits are not linear with respect to the data. Therefore fitting individual subject data allowed us to make unbiased estimates of average patient group generalization and compliance functions. Additionally, fitting individual subject data allowed us to make direct estimates of error bounds for the average fits without resorting to bootstrapping techniques which can be problematic for smaller data sets.

This model was fit to the data from each subject in each group to estimate the compliance and error generalization parameters for that individual. In the preceding equations the parameters $D$ and $B$ are eight-dimensional vectors (1 dimension for each direction of movement). $D$ represents the compliance (inverse of stiffness) in each movement direction, whereas $B$ represents the generalization magnitude for differences in movement direction between -180 and $+180^{\circ}$. To reduce the number of parameters, we noted that arm compliance in the horizontal plane is well modeled by a $2 \times 2$ compliance matrix (Mussa-Ivaldi et al. 1985). We derived the relationship between this two-dimensional compliance matrix and the direction-dependent onedimensional compliance in our model and found that the latter depended only on the symmetric components of the former (see APPENDIX 1). Therefore we constrained $D$ to three free parameters according to this relationship. Furthermore, previous work has shown that error generalization functions are essentially symmetric (i.e., the amount of generalization to $-45^{\circ}$ is essentially the same as generalization to $+45^{\circ}$ ). Therefore we enforced symmetry on $B$, which constrained it to five free parameters.

Because the force-field was kept constant on all trials in which it was applied but the actual force experienced varied depending on the movement velocity, we followed Thoroughman and Shadmehr (2000) in representing the force input to the model, $f(n)$, as a discrete scalar representing force-field magnitude and assigning it values of 1,0 , or -1 corresponding to field A, catch, or field B trials, respectively. Thus the compliance computed from this model has units of angular error, and it corresponds to the aiming error directly induced by the forcefield. Although this measure of compliance is nonstandard, it is intrinsically related to the usual measure of compliance, and the relationship between the two is a simple multiplicative constant (see APPENDIX 2). We use this conversion to report stiffness in Fig. 5.

We used a least-squares optimization to find the parameters, $B$ and $D$, that minimized the difference between the aiming error sequence predicted by the model and the actual aiming errors produced by each subject.

\section{RES ULT S}

Subjects initially reached in a null field, then in field A (a clockwise curl field), and finally in field B (a counter-clockwise curl field). Figure 1 plots hand paths for typical subjects. The force field perturbed movements (Fig. 1, 2nd row), but reaching of control subjects improved with training (Fig. 1, 3rd row), and errors in catch trials closely mirrored the errors made on initial exposure to the field. Subjects with HD also appeared to learn the field. Like controls, initial exposure to the field substantially perturbed movements in HD, but their movements late in training trials became straighter and their catch trials closely mirrored the errors recorded in field trials. However, stemming from their feedback control dysfunction (Smith et al. 2000), HD subjects showed some difficulty stopping their movements. In contrast, subjects with cerebellar degeneration showed signs of impaired adaptation. In catch trials, movements did not have errors that mirrored the errors in field trials, indicating that these subjects did not produce anticipatory compensation for the field. 


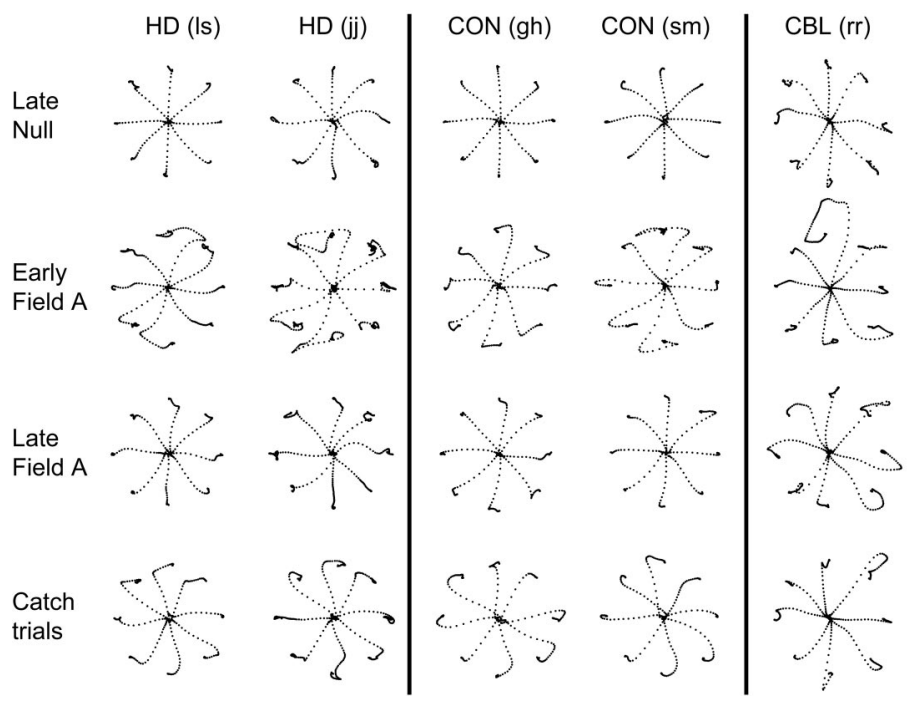

Errors in the null field

Our task involved holding a robot with potentially significant passive dynamics. Therefore even in the null field sets the robot's passive dynamics could contribute to reaching errors (Fig. 2A). These errors would in turn require an adaptive response. We noted that despite training, the cerebellar subjects still displayed substantial errors in the null sets (Fig. 2B). We began by asking whether these errors were related to the passive dynamics of the robot.

The robot's passive dynamics produced forces on the hand that depended on movement direction. To explore the effect of these forces on reaching, we simulated dynamics of the human arm coupled to our robot and noted the trajectory errors when the human arm controller did not account for the robot's dynamics. We computed the error in direction of motion at 300-ms movement time for each target direction. Counter clockwise errors were marked as positive, and clockwise errors were marked as negative. The average error across target directions had a zero mean, but for each target direction, the error was consistently positive or negative (thick black lines in Fig. 2C). For example, the simulations predicted that robot's

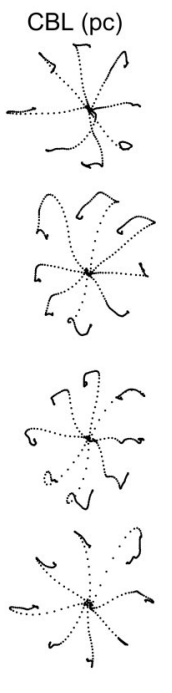

FIG. 1. Hand paths from representative subjects. Subject groups: HD, symptomatic HD; CON, controls; CBL, cerebellar degeneration. Movement sets: null, final movement in each direction in the null field; early $\mathrm{F}$, first movement in each direction after onset of field $\mathrm{A}$; late $\mathrm{FF}$, last movement in each direction during final set of field $\mathrm{A}$; catch trials, last catch trial in each direction during final set of field A.

passive dynamics should produce counter clockwise errors for reaches toward $45^{\circ}$ and clockwise errors for reaches at $135^{\circ}$.

Reach trajectories of a control subject and a cerebellar subject in the first null set (averaged for each direction) are shown in Fig. 2A. To compare the direction-dependent pattern of errors with the errors produced by the model, Fig. $2 C$ plots average error as a function of direction. The model predicted positive (counter clockwise) errors for targets at 45 and $225^{\circ}$, and negative errors for targets at 135 and $315^{\circ}$. This pattern is present in the reaching trajectories of the two subjects plotted in Fig. 2A. Average errors across subjects in each group also tended to show a direction dependent pattern of errors similar to the model (Fig. 2C). However, the directional errors tended to be smaller than predicted for targets at 135 and $315^{\circ}$ and larger than predicted for targets at 45 and $225^{\circ}$.

With training in the null set, control and HD subjects produced significantly reduced direction-dependent errors (Fig. $2 E, P<0.01$ for control, pre-HD, and HD groups). Figure $2 D$ shows that errors in these groups converged toward the middle circle (the middle circle indicates 0 error in each direction). However, cerebellar subjects continued to produce errors that

A

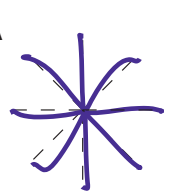

Control subject Early null (1st set)

C

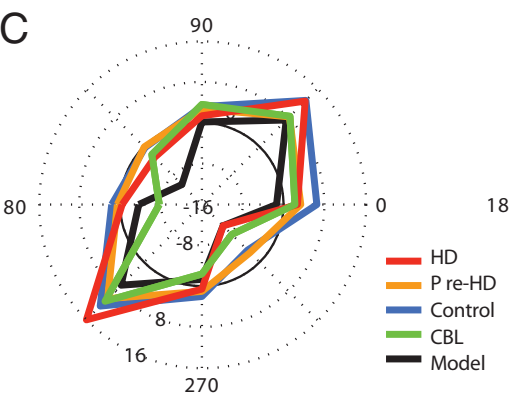

Early null (1st set)

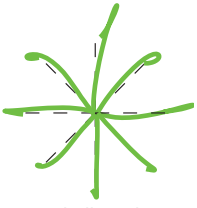

Cerebellar subject Early null (1st set)

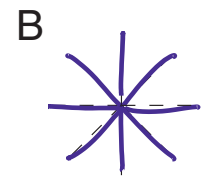

Control subject Late null (4th set)

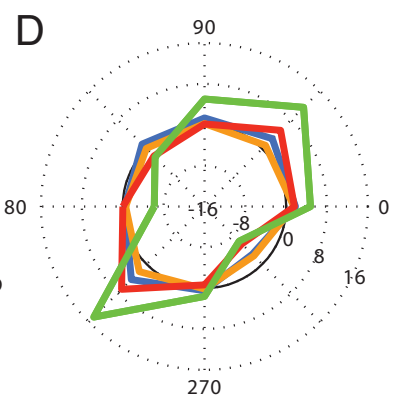

Late null (4th set)

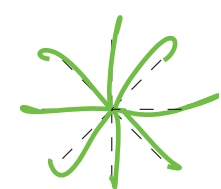
Cerebellar subject
Late null (4th set)
E

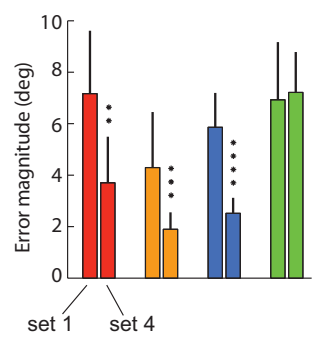

FIG. 2. Analysis of aiming errors in null field sets. $A$ and $B$ : average hand paths in the 1st set and in the 4th set of null field trials for a control and a cerebellar subject. $C$ and $D$ : aiming errors (at 300-ms movement time) are plotted as a function of movement direction in the 1 st and last null field sets. The middle black circle denotes 0 error. Counter clockwise errors are marked positive and are plotted outside of this circle and clockwise errors are marked negative and are plotted inside of the circle. $E$ : change in magnitude of error with null field training. The average magnitude of error across all movement directions is shown for the 1st and last null field sets. HD and control groups display significant reductions in directional error from the first to the last set $(P<0.01$ for all groups). There is no significant change in the cerebellar group $(P>0.5)$. 
were essentially unchanged throughout the null field training (Fig. 2E). Therefore it appeared that although the passive dynamics of the robot was a significant factor in the errors that all subjects produced in the null field training sets, with training all but cerebellar subjects were able to adapt to the novel dynamics.

\section{Errors in the force fields}

Figure 3 plots movement errors for each group in all the null and field sets (bin size $=\sim 25$ trials). In control subjects, training produced a reduction in errors in field trials and an increase in errors in catch trials. When we switched the field from A to B, large errors were produced in field trials, but these errors declined rapidly. Similarly, catch trial errors changed sign and became appropriate for field $\mathrm{B}$. If motor commands compensated for $50 \%$ of the forces in a field, then errors in catch trials should be equal in magnitude but opposite in direction to errors in field trials. By the third set in field A, for control subjects, the catch trial errors were more than double the field trial errors. To estimate percent compensation of the field, we computed a learning index that considered errors in catch and field trials. The index is 1 if there is $100 \%$ compensation for field A, -1 if there is $100 \%$ compensation for field $\mathrm{B}$, and 0 if compensation is appropriate for a null field. With $1 / 6$ probability of catch trials, maximum possible compensation is $83 \%$. Figure $3 B$ shows that in control subjects, three sets of training produced $\sim 65 \%$ compensation for the field.
Presymptomatic and symptomatic HD subjects displayed similar behavior to controls. Field trial errors decreased with training while catch trial errors increased (Fig. 3A). By the 3rd set of training in field A, both groups of HD subjects had catch trial errors that were generally larger than field trial errors. This implies $>50 \%$ compensation for the field. The learning indices were very similar between the HD and control groups in both field A (Fig. 3, $B$ and $D$ ) and field B (Fig. 3B). Size of errors in catch trials was not significantly different in HD and control subjects (Fig. 3, $B$ and $E$ ). Percent improvement in force-field trial errors was comparable in HD and control subjects (Fig. 3, $B$ and $F$ ). Therefore the data suggested that the HD subjects adapted normally to both the passive and active force fields produced by the robot.

By the third set of training in field A, errors in field trials were not significantly different between the cerebellar subjects and other groups (Fig. 3A). However, the cerebellar subjects displayed significantly smaller errors in catch trials (Fig. $3 E, P<0.001$ compared with controls and $P<0.01$ compared with symptomatic HD subjects) and significantly smaller learning indices (Fig. $3 D, P<0.0001$ compared with controls and $P<0.01$ compared with symptomatic HD subjects). It is possible that in cerebellar subjects, the field trial errors were relatively small because of increased stiffness of the arm. The lack of field-dependent errors in catch trials seems consistent with this. For example, the $y$ axis of Fig. $3 C$ plots the change in catch trial errors for each subject when the field switched from A to B.
A
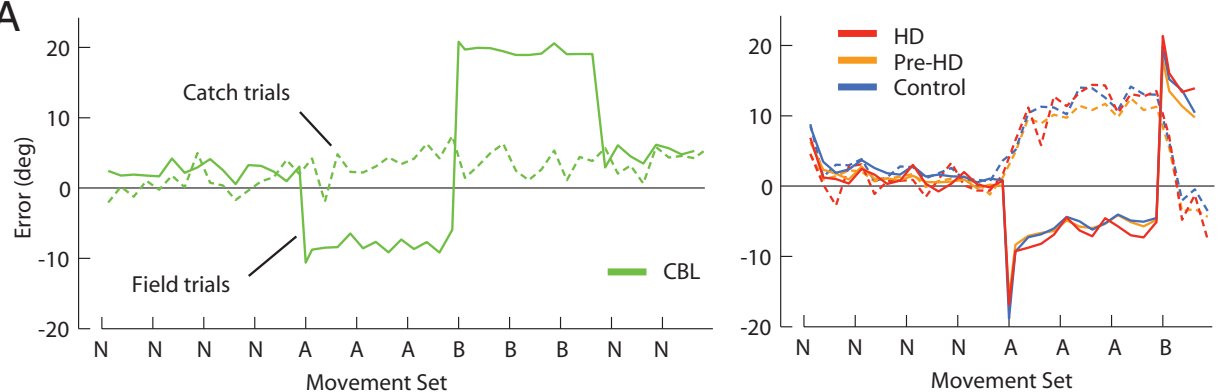

B
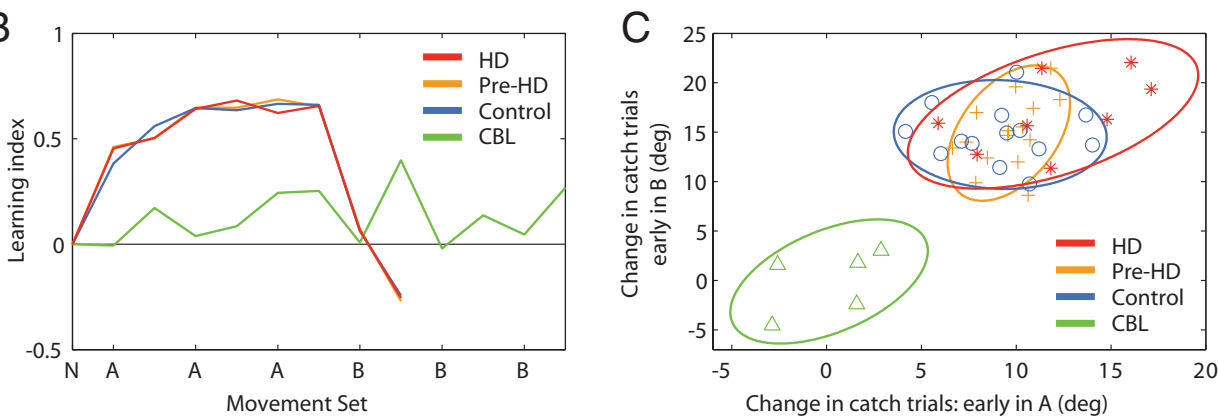

D

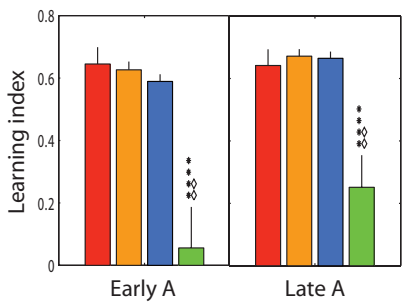

E

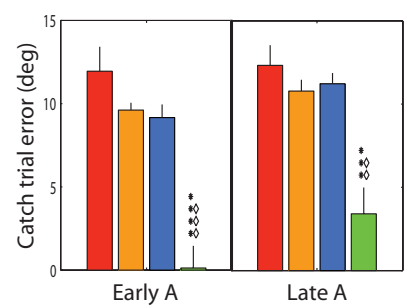

$\mathrm{F}$

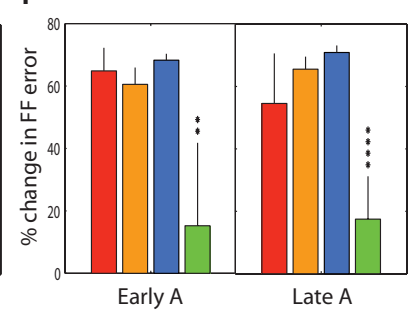

FIG. 3. Analysis of aiming errors in the force field sets. $A$ : aiming errors as a function of target sets. Positive values indicate clockwise errors with respect to target direction. The $x$ axis denotes the applied field. $\mathrm{N}$, null. $A$ : clockwise curl field; $B$ : counter-clockwise curl field. Each set contained 96-100 movements. There are 4 bins per target set $(\sim 21$ field trials and 4 catch trials per bin). In the null field sets, there were no true field or catch trials, but the movement numbers corresponding to these during the field sets were designated as field or catch trials for the sake of comparison. $B$ : learning index indicates the ratio of errors in catch and field trials (see METHODS) and is an estimate of the percent compensation for the field. A value of 1 indicates full compensation for field $\mathrm{A}$ and -1 indicates full compensation for field $\mathrm{B}$. $C$ : within subject changes in catch trial errors for all subjects in the study. The $x$ axis indicates change in catch trial errors in early field A (set 1) vs. null field. The $y$ axis indicates change in catch trial errors in field B (set 1) vs. late field A (set 3). The ellipses represent $95 \%$ confidence areas for each group. $D-F$ : measures of performance during 1st and 3rd training sets in field A (mean \pm $\mathrm{SE}$ ). Data from the 1st training last bin of the first training set are labeled "early A," and the data from the last bin in the 3rd training set are labeled "late A." $D$ : learning index. $E$ : catch trial errors. $F$ : percent change in field trial errors. *, significant differences from controls; $\diamond$, significant differences from subjects with manifest HD. $* P<0.05$, $* * P<$ $0.01, * * * P<0.001$, *****P<0.0001. All $P$ values are corrected for multiple comparisons. 
A

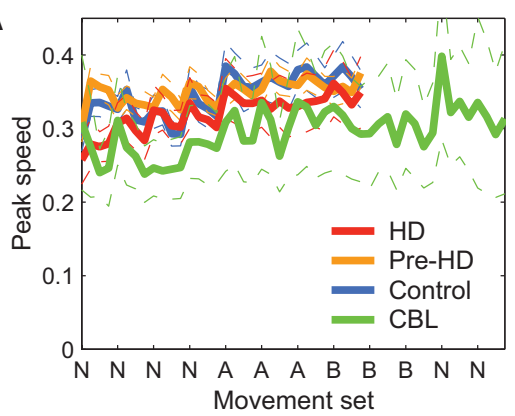

C

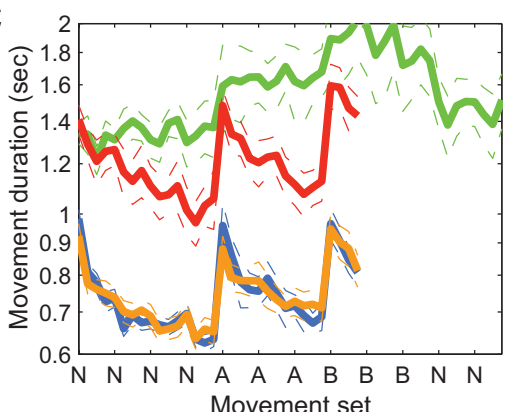

E

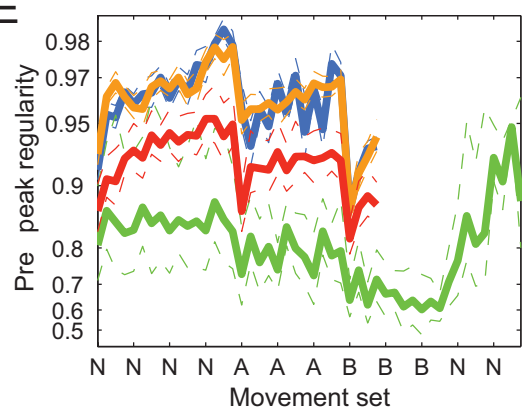

B

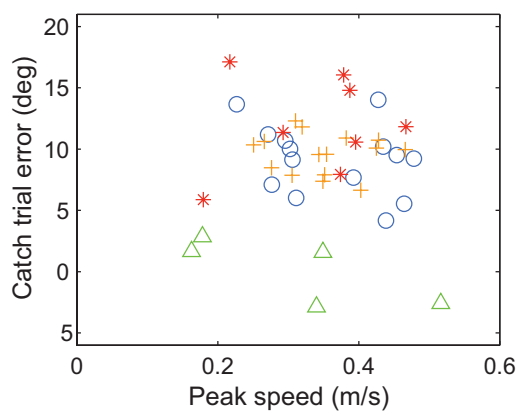

D

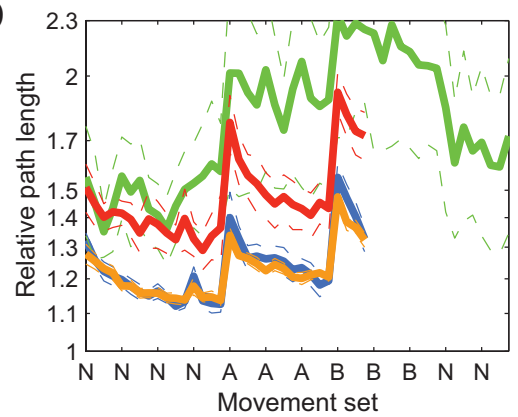

$\mathrm{F}$

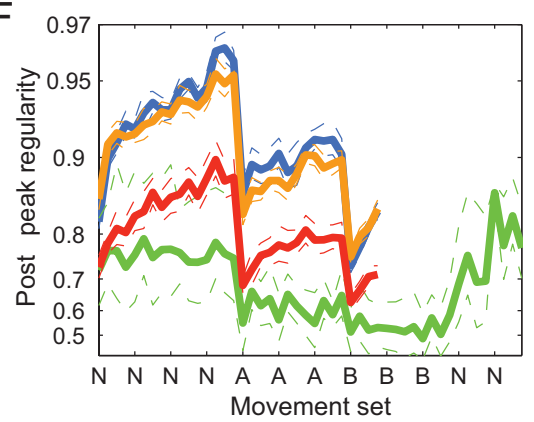

FIG. 4. Movement speed, movement duration, movement regularity, and relative path lengths. $A$ : time course of peak speed during movement. $B$ : the relationship between peak speed and catch trial error. The data are for the trials in the first set of field A (size of catch trial is computed with respect to the last null set). $C$ : time course of movement duration (data for catch trials are not shown). Movement duration is the time elapsed between movement onset and movement termination which are determined by a velocity threshold of $3 \mathrm{~cm} / \mathrm{s}$ (see METHODS). $D$ : time course of relative path length in field trials (data for catch trials are not shown). Relative path length is the path length for each trial divided by the straight line distance between start and endpoints. $E$ and $F$ : time course of movement regularity for the 1 st and 2nd halves of each movement, respectively. The halfway point of each movement is defined as the time point at which the peak speed occurs. Movement regularity for each segment is defined as the correlation coefficient between the velocity profile of that segment and that of the most typical segment for the corresponding target direction in the null field trials.
The $x$ axis of Fig. $3 C$ plots the change in catch trial errors when the field switched from null to A. In HD and control groups, catch trial errors in every subject changed significantly when the field switched from null to A and then to B. This was not the case for the cerebellar subjects. Furthermore, while control and HD subjects displayed $60-70 \%$ reduction in errors in field trials (Fig. $3 F$ ), cerebellar subjects displayed a significantly smaller error reduction of $10-20 \%(P<0.01)$. Therefore the data suggest that subjects with cerebellar degeneration displayed an impaired ability to alter their motor commands to compensate for the applied force fields.

As a group, cerebellar subjects reached $\sim 15 \%$ more slowly (Fig. 4A) than controls, and subjects with HD reached with speeds intermediate between cerebellar subjects and controls. Size of catch trial errors might be expected to be larger in movements that are faster. Could the difference in movement speeds explain the difference in the size of errors in catch trials? To explore this possibility, we compared within subject relationships between movement speed and size of catch trial errors. We found that catch trial errors were not modulated with movement speed over the speed range present in this experiment (Fig. 4B). Cerebellar subjects displayed small catch trial errors at slow, midrange, and fast speeds. In contrast, HD and control subjects displayed large catch trial errors over the same range of movement speeds.
We also considered movement parameters other than aiming errors. Figure 4, $C$ and $D$, plots movement times and path lengths. As the task began in the null field, the HD and cerebellar subjects had nearly identical movement times and path lengths. Whereas in HD subjects training produced a reduction in this variable, no changes were apparent in the cerebellar group. When the field was introduced, movement time increased in all subjects, but failed to decline with practice in the cerebellar group. Similar patterns were evident in movement path lengths (Fig. 4D). Therefore measures of task performance other than aiming errors also suggested normal adaptation in symptomatic and presymptomatic HD subjects but impaired adaptation in cerebellar subjects.

\section{Trial-to-trial effect of error}

Figure $5 \mathrm{~A}$ shows the time course of movement errors for a single direction $\left(90^{\circ}\right)$ during training in field A for control subjects. For clarity, errors for intervening trials that were not in this same direction are not shown. A steady-state pattern is reached after $\sim 50$ total trials - this corresponds to $\sim 5-8$ trials in each movement direction. However, note that the force-field movement after each catch trial generally shows an increase in error magnitude relative to the movement before that catch trial, whereas between catch trials, errors on force-field movements are reduced. In Fig. 5B, we aligned the sequence of 
A

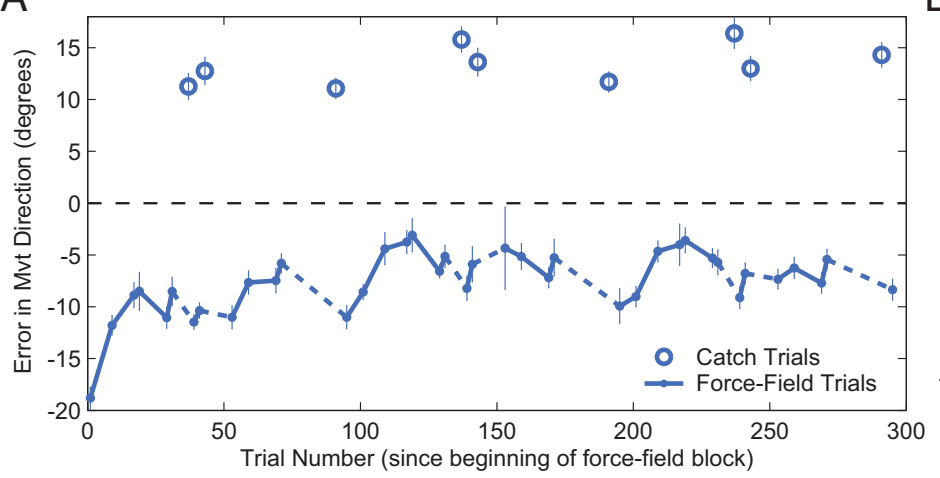

B

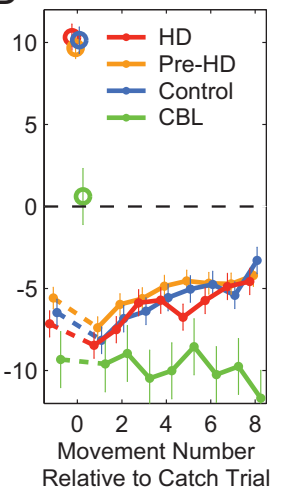

C

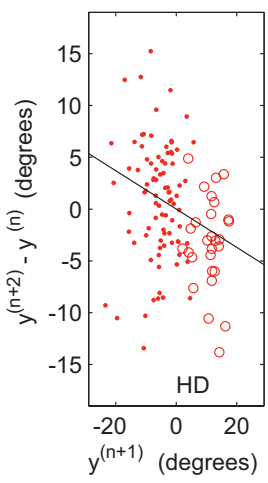

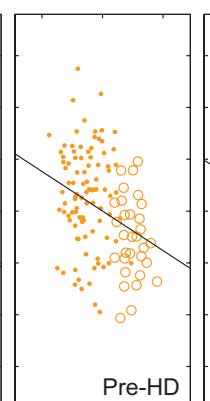

Pre-HD

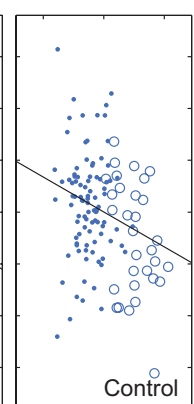

Control

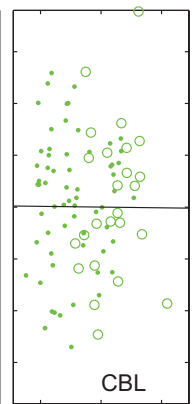

D

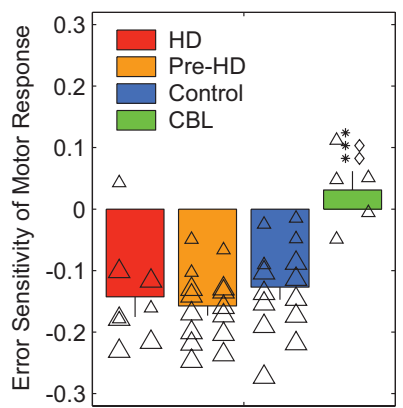

E

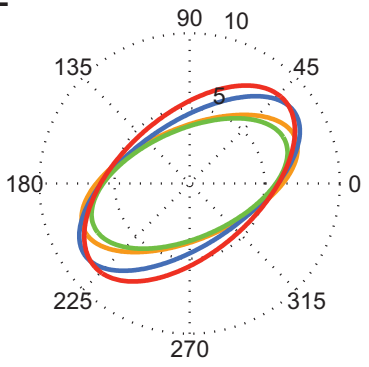

$\mathrm{F}$

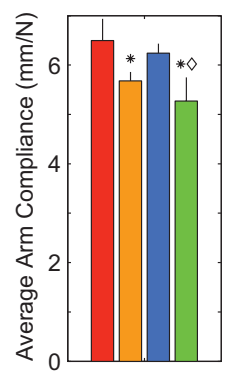

G

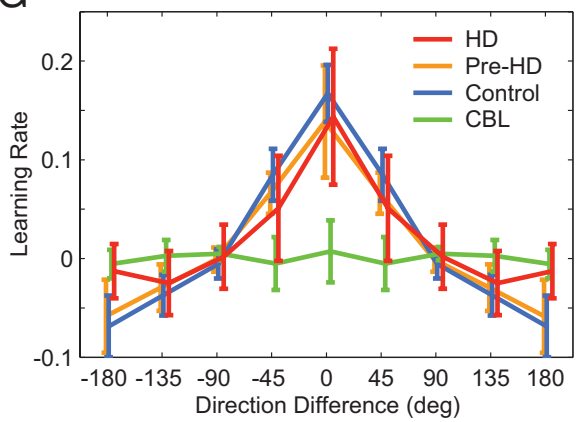

FIG. 5. Analysis of trial-to-trial error-dependent learning. A: errors for all trials in field $\mathrm{A}$ for reaches toward $90^{\circ}$ averaged across the control subjects. The $x$ axis represents trial number for movements in all directions and so the horizontal spacing between consecutive $90^{\circ}$ movements indicates the number of intervening trials in other movement directions. Force-field trials are shown as filled dots and catch trials are represented as open circles. Consecutive force-field trials are connected by solid lines, whereas the force field trials before and after a catch trial are connected by a dotted line. Note the saw-tooth pattern of errors on force field trials where the error increases in magnitude after catch trials (dotted lines) then generally decreases after force-field trials. $B$ : response to catch trial errors. All trials in field A for a given target direction were aligned to catch trials and were averaged across directions and across subjects. In all groups except cerebellar, error in the trial that followed a catch trial appeared larger in magnitude than error in the trial that preceded the catch trial. After the catch trial, errors declined in the control and HD groups, whereas cerebellar subjects showed little or no change in errors. $C$ : trial-by-trial response to errors for typical subjects in each group. Data from force-catch-force (FCF) and force-force-force (FFF) triplets were analyzed. The change in error between the 1st and 3rd movements is plotted against the error in the 2nd movement in each triplet. FFF triplets are plotted as filled dots and FCF triplets as open circles. Because the 1st and 3rd trial share the same force-field environment, the change in error from the 1st to the 3rd trial is interpreted as a change in motor output. Therefore this plot illustrates the relationship between motor errors and subsequent changes in motor output. For HD and control subjects, there is a strong negative relationship between middle movement error and the subsequent change in motor output. This negative relationship indicates an adaptive change in motor output that would reduce error in the middle movement were that movement type repeated. The slope of the best fit straight line through the data provides, for each subject, an estimate of the sensitivity of motor output change to motor error. $D$ : comparison of error sensitivity between groups. The bar graph indicates mean sensitivity for each group, and error bars indicate means \pm SE. The triangular symbols show sensitivity estimates for individual subjects. Large triangles indicate subjects for which the individual sensitivity estimate was significantly negative. Note that as a group, CBL subjects display a significantly decreased magnitude of error sensitivity compared with control and HD subjects. The average CBL subject error response is not significantly different from 0 (and nominally in the wrong direction for reducing middle movement errors). $E-G$ : results for state-space model of trial to trial learning. This model makes estimates of the sensitivity of motor put change not only to errors in that same movement direction (as in the triplet analysis) but also to errors in other movement directions (see METHODS). $E$ : estimated compliance ellipses for the arm. This plot shows the direction-dependent pattern of compliance for each group. $F$ : comparison of average compliance across groups. $G$ : comparison of error generalization functions. In $D$ and $F$, asterisks indicate significant differences from the control group. Diamonds indicate significant group differences from subjects with manifest HD. One asterisk, $P<0.05 ; 2$ asterisks, $P<0.01 ; 3$ asterisks, $P<0.001 ; 4$ asterisks, $P<0.0001$. 
movement errors to catch trials and averaged the results across subjects and across all movement directions. This analysis shows that in healthy subjects field trial errors increase in magnitude following catch trials and decrease after field trials. The resulting saw-tooth pattern of error that can be seen in Fig. $5 A$ has been attributed to error-dependent trial-to-trial learning (Donchin et al. 2003; Thoroughman and Shadmehr 2000) in which catch trial errors lead to partial unlearning of the field and force-field trial errors lead to partial re-learning of the force-field. Unlike the control and HD subjects, cerebellar subjects had essentially zero error in catch trials and displayed no improvement in the subsequent field trials suggesting reduced error-dependent learning.

To quantify the amount of trial-to-trial learning, we assessed the relationship between error on a given trial and the adaptive motor response to that error on a subsequent trial. To accomplish this, we compared all movement triplets in each target direction that were FCF or FFF. Error in the first movement is labeled $y^{(n)}$; second movement is $y^{(n+1)}$, etc. Note that for a given direction, $y^{(n+1)}$ is the next movement after $y^{(n)}$, but there may be intervening trials in other directions. Figure $5 C$ plots $y^{(n+2)}-y^{(n)}$ as a function of $y^{(n+1)}$ for all FCF and FFF triplets in each target direction for each subject. For each subject, we computed the slope of a line through this data and then computed an average slope across subjects in that group. This slope represented the average sensitivity of motor output to error in the previous trial. An adaptive response to error should display a negative slope. Average sensitivity to error is plotted for each group in Fig. 5D. HD and control subjects displayed significantly negative error sensitivities $(\mathrm{HD}=$ $-0.13, P=0.003$; pre-HD $=-0.15, P=0.0003$; control $=$ $-0.15, P=0.0008)$, but cerebellar subjects had a sensitivity close to zero (CBL $=+0.04, r=0.06, P=0.8$ ). Therefore in all groups except cerebellar, the change in movement errors from $y^{(n)}$ to $y^{(n+2)}$ was related to the error $y^{(n+1)}$.

Figure $5 C$ also shows that in cerebellar subjects, there was a variance in the measure $y^{(n+2)}-y^{(n)}$ that was comparable to other groups. Therefore the motor commands in cerebellar subjects did vary from trial to trial. However, these trial-to-trial changes were uninformed by the error in the previous trial.

The pattern of errors in Fig. $5 \mathrm{~A}$ is partially but not fully explained by the average responses to same-direction catchtrial errors shown in Fig. 5B. Thoroughman and Shadmehr (2000) demonstrated that much of the deviation more this average response can be explained by the effect of errors experienced in other movement directions. The triplet analysis described in the preceding text assessed the trial-by-trial effect of error by relating motor output changes in a certain movement direction to errors experienced only in that same direction. However, errors made in other movement directions may also influence motor output changes in a given direction, and so a more general analysis of the trial-to-trial effect of error could consider all of the interactions between errors made in one direction and motor output changes in another. This pattern of interactions has been termed generalization. We estimated the pattern of generalization of error-dependent learning from one movement direction to another using a state-space model of direction-dependent trial-to-trial learning (see METHODS). Here the idea is that when an error is experienced in a certain movement direction, subsequent movements in that direction are programmed differently to partially compensate for the error experienced. But subsequent movements in other directions may also be modified because of that error experience. The generalization pattern of error describes this effect by quantifying the amount of compensation induced by error as a function of the directional similarity between the trial on which a given error was experienced and subsequent trials on which this compensation manifests.

The state-space model we used is similar to several other autoregressive models of trial-to-trial motor adaptation used to study motor in healthy individuals. (Donchin et al. 2003; Scheidt et al. 2001; Thoroughman and Shadmehr 2000). It determines arm compliance and the pattern of error generalization for each subject by finding the values of these parameters that best predict the sequence of errors produced by that subject. Arm compliance (the inverse of stiffness) and generalization patterns of error are shown for all groups in Fig. 5, $E-G$. Compliance varies substantially from one direction of motion to another because of the biomechanical properties of the arm. Figure $5 E$ shows that the size and shape of the compliance ellipses are similar for all groups. Cerebellar subjects and presymptomatic HD subjects do display somewhat smaller average compliance (greater stiffness) than other groups (see Fig. $5 F$ ), but all groups display average compliance within $15 \%$ of control values.

Figure $5 G$ shows that the healthy volunteers and HD subjects that we tested display a pattern of generalization similar to that previously reported for normal controls (Thoroughman and Shadmehr 2000). For HD and control subjects, directional error generalizes with a significantly positive learning rate to movements in the same direction (directional difference $=0$ ), the strength of this generalization falls to $\sim 50 \%$ at $45^{\circ}$, and becomes negative for movements in the opposite direction (directional difference $=180^{\circ}$ ). CBL subjects show a markedly different pattern. They fail to show any consistent generalization of error from one movement direction to another, suggesting that errors experienced on one trial fail to influence motor output on subsequent trials.

\section{I S C U S S I O N}

We altered the dynamics of reaching movements by imposing passive and active force fields on the hand. These forces produce reaching errors. It is thought that the errors modify an internal model with which the brain computes motor commands that initiate a reach. We found that this ability was intact in asymptomatic individuals at risk for HD as well as patients with manifest HD. In contrast, patients with cerebellar degeneration were profoundly impaired. Whereas in control and HD groups, the errors in a given trial produced compensatory changes in the motor commands that initiated the next trial, in cerebellar patients, the changes in motor commands were unrelated to the error in the previous trial. Furthermore, a quantitative model of error-dependent learning showed that HD subjects not only learned normally from error but also generalized what they learned from one direction to another with the same spatial pattern as healthy controls. In contrast, cerebellar patients displayed substantial deficits in both trialto-trial adaptation and generalization.

Symptomatic HD individuals that we studied here were clinically classified as mild to moderately affected in their movements [median quantified neurological examination 
(QNE) score of 27, see METHODS]. MRI analysis suggests that when the clinical symptoms are detected, the mildly affected individuals have significant loss of volume in the caudate and putamen (Harris et al. 1996) with the damage to the putamen more prominent than the caudate. Examination of brain regions other than basal ganglia in mildly affected individuals (QNE $<$ 41) has not found any significant differences in the volumes of the frontal, parietal, occipital, and temporal lobes, or the volumes of the cerebellum and the brain stem, despite having found clearly abnormal basal ganglia volumes (Aylward et al. 1998). Taken together, it is likely that the HD genetic mutation in the individuals that we studied had resulted in atrophy of the striatum, in particular the putamen, although the possibility of mild cortical damage cannot be excluded.

Both the cerebellum and striatum have been implicated as neural structures that contribute to motor control and learning, yet the contribution of each is not well understood. The learning deficit that we found in cerebellar subjects confirms a previous finding that the learning of internal models of limb dynamics depends on the integrity of the cerebellum (Maschke et al. 2004). In this work, a group of patients with cerebellar atrophy secondary to SCA-6 showed decreased force-field adaptation. Our finding extends that work by showing that this deficit in cerebellar patients is related to a lack of sensitivity of motor commands to errors in the previous trial and by demonstrating that individuals with cerebellar damage from a variety of etiologies can show marked deficits in force-field adaptation.

Our observation that learning of internal models of physical dynamics is impaired in cerebellar subjects is consistent with the functional imaging results that have suggested a role for the cerebellum in this kind of learning (Nezafat et al. 2001). It is also consistent with the finding that the learning of internal models of dynamics relies on motor primitives that have receptive field properties similar to those reported for some Purkinje cells in the cerebellum (Donchin et al. 2003) and that Purkinje cells in the cerebellum display simple spike activities that correlate with anticipation of predictable load force perturbations during grasping (Dugas and Smith 1992).

However, It has been suggested that habit formation (often loosely defined as stimulus-response behavior) and skill learning in general (Bachevalier 1990; Mishkin and Appenzeller 1987; Mishkin et al. 1984; Paulsen et al. 1993), or more specifically open-loop skill learning, is disturbed in HD and with striatal dysfunction. Our results are inconsistent with this generalization. Because of sensorimotor feedback delays (Cordo 1990; Miall et al. 1986), the first 200-300 ms of movement is open-loop. Data taken from this open loop period in the force-field task show normal learning by HD subjects, indicating that at least certain kinds of skill learning, and in particular open-loop skill learning, can be well-preserved in both symptomatic and presymptomatic carriers of the HD gene. The preservation of open-loop skill learning in HD is supported by a recent study demonstrating intact prism adaptation despite somewhat decreased aftereffects in a large sample of patients with HD and Parkinson's disease (FernandezRuiz et al. 2003).

Our results that in HD there is an intact ability to adapt to altered dynamics of reaching movements is consistent with the finding that in HD there is also an intact ability to adapt to altered kinematics of reaching movements (Fernandez-
Ruiz et al. 2003). Remarkably, cerebellar damage can profoundly inhibit learning in both of these conditions (Chen et al. 1996; Martin et al. 1996; Maschke et al. 2004; Robertson and Miall 1999; Sanes et al. 1990; Topka et al. 1993). Computationally, this kind of learning involves using the errors in a movement to update the sensorimotor map that transforms the desired behavior (or limb states) into motor output that produces that movement (i.e., the inverse model). In other words, errors are associated with the recent history of sensory states of the limb.

In contrast, HD patients typically perform poorly on tasks that depend on an ability to produce reactions to real-time sensory input. For example, HD patients are impaired in learning the pursuit rotor task where limb movements try to follow a repeating goal trajectory lasting 30-60 s (Willingham et al. 1996). That task depends substantially on the incorporation of real-time sensory information about the current trajectory to correct and minimize errors between the actual and desired trajectory.

Our earlier work suggesting that this error feedback system is disturbed in HD (Smith et al. 2000) is consistent with several lines of evidence that point to disordered responses to real-time sensory feedback as a manifestation of HD. Reaction times to both auditory and visual stimuli are slowed even early in the disease course (Kirkwood et al. 2000; Siemers et al. 1996). The cortically mediated (Marsden et al. 1978; Petersen et al. 1998; Rothwell 1990) long-latency component of the stretch reflex responses is reduced or absent in HD patients (Noth et al. 1985; Thilmann et al. 1991; Thompson et al. 1988), and electrical potentials evoked in somatosensory cortex by peripheral nerve stimulation are reduced throughout the course of HD (Meyer et al. 1992; Topper et al. 1993). Additionally, the modulation of manual grip forces when grasping objects has also been found to be disturbed in HD (Fellows et al. 1997; Schwarz et al. 2001). Taken together, it might appear that a generalized dysfunction in the processing of sensory information occurs in HD.

However, our present findings are sharply inconsistent with this characterization. Instead, our results suggest that in HD, there is an intact ability of the motor system to respond to sensed errors from movement-to-movement but a reduced ability to form an appropriate response during the execution of the movement. Therefore in HD the deficits in reaching cannot be due to a general deficit in sensing movement errors. Rather, the sensory processing deficits may be limited to the on-line utilization of sensory information and the on-line feedback control of the limb in response to error. Conversely, we see that with degeneration of the cerebellum the ability to adjust motor output from trial to trial in response to error is impaired despite intact ability to produce in-flight error-correcting responses, suggesting distinct neural bases for these two important responses to error. In computational terms, these observations hint that in $\mathrm{HD}$, deficits in simple reaching may be related to monitoring movement progress and producing real-time responses to sensed error. In cerebellar degeneration, the deficits in simple reaching may be related to dysfunctional updating of internal models that transform high level kinematic movement plans into motor commands. 
APPENDIX 1: RELATIONSHIP BETWEEN TWO-

DIMENSIONAL COMPLIANCE AND ONE-

DIMENSIONAL OPPOSITIONAL COMPLIANCE

To translate a two-dimensional compliance matrix into the effective one-dimensional oppositional compliance for each direction of movement, we derived the relationship between these variables. The twodimensional compliance matrix is defined by

$$
\left[\begin{array}{l}
x \\
y
\end{array}\right]=\left[\begin{array}{ll}
D_{11} & D_{12} \\
D_{21} & D_{21}
\end{array}\right]\left[\begin{array}{l}
F x \\
F y
\end{array}\right]
$$

In our experiment, both the perturbing force-field and the error measure are in the same direction on any given trial (the direction perpendicular to movement). So the effective one-dimensional compliance can be defined as the constant of proportionality between the projection of the two-dimensional displacement onto the two-dimensional force and the magnitude of the two-dimensional force, $F$. Because this one-dimensional compliance may depend on the direction of the applied force we will refer to it as $D_{1}(\partial)$

$$
\text { (projection of } \left.\left[\begin{array}{l}
x \\
y
\end{array}\right] \text { onto }\left[\begin{array}{l}
F x \\
F y
\end{array}\right]\right)=D_{1}(\partial) \cdot F
$$

Note that the value of the projection of a vector A onto another vector $\mathrm{B}$ can be computed as the dot product of $\mathrm{A}$ and a unit vector in the direction of $\mathrm{B}$. If we use this relation and we represent the applied force in terms of its magnitude $F$ and direction $(\partial)$, then the preceding equation becomes

$$
\frac{\left[\begin{array}{l}
x \\
y
\end{array}\right] \bullet\left[\begin{array}{c}
F \cdot \cos \partial \\
F \cdot \sin \partial
\end{array}\right]}{F}=D_{1}(\partial) \cdot F
$$

substituting from $E q$. Al yields

$$
\frac{\left(\left[\begin{array}{ll}
D_{11} & D_{12} \\
D_{21} & D_{21}
\end{array}\right]\left[\begin{array}{l}
F \cdot \cos \partial \\
F \cdot \sin \partial
\end{array}\right]\right) \cdot\left[\begin{array}{c}
F \cdot \cos \partial \\
F \cdot \sin \partial
\end{array}\right]}{F}=D_{1}(\partial) \cdot F
$$

this simplifies to

$$
\left(\left[\begin{array}{ll}
D_{11} & D_{12} \\
D_{21} & D_{21}
\end{array}\right]\left[\begin{array}{c}
\cos \partial \\
\sin \partial
\end{array}\right]\right) \cdot\left[\begin{array}{c}
\cos \partial \\
\sin \partial
\end{array}\right]=D_{1}(\partial)
$$

$D_{11} \cos ^{2} \partial+D_{12} \cos \partial \sin \partial+D_{21} \cos \partial \sin \partial+D_{22} \sin ^{2} \partial=D_{1}(\partial)$

$\frac{D_{11}+D_{22}}{2}+\left(\frac{D_{11}-D_{22}}{2}\right) \cos (2 \partial)+\left(\frac{D_{12}+D_{21}}{2}\right) \sin (2 \partial)=D_{1}(\partial)$

without loss of generality we can rewrite the off-diagonal elements of the compliance matrix as

$$
D_{12}=D_{\mathrm{S}}+D_{\mathrm{A}} \text { and } D_{21}=D_{\mathrm{S}}-D_{\mathrm{A}}
$$

where $D_{\mathrm{S}}$ and $D_{\mathrm{A}}$ are the symmetric and anti-symmetric off-diagonal components of the two-dimensional compliance matrix

$$
\left[\begin{array}{cc}
D_{11} & D_{12} \\
D_{21} & D_{21}
\end{array}\right]=\left[\begin{array}{cc}
D_{11} & D_{\mathrm{S}}+D_{\mathrm{A}} \\
D_{\mathrm{S}}-D_{\mathrm{A}} & D_{21}
\end{array}\right]
$$

Substituting into the previous equation yields

$$
\frac{D_{11}+D_{22}}{2}+\left(\frac{D_{11}-D_{22}}{2}\right) \cos (2 \partial)+D_{\mathrm{S}} \cdot \sin (2 \partial)=D_{1}(\partial)
$$

Note that $D_{1}(\partial)$ depends on $D_{11}, D_{22}$, and $D_{\mathrm{S}}$ but not on $D_{\mathrm{A}}$. This implies that the amount of asymmetry in the two-dimensional compliance matrix has no effect on the one-dimensional compliance. Conversely, the amount of asymmetry in the two dimensional compliance cannot be determined from characteristics of the one-dimensional compliance. Note also that $D_{1}$ has $3 \mathrm{df}$ and is a function of $\partial$, meaning that the magnitude of the one-dimensional compliance depends on the direction of the applied force but can always be fully described by three parameters $D_{11}, D_{22}$, and $D_{\mathrm{S}}$ as shown in the last equation above. In the state-space learning model, $D_{1}(\partial)$ was constrained according to this equation.

APPENDIX 2: RELATIONSHIP BETWEEN REAL COMPLIANCE AND THE COMPLIANCE MEASURE INTRINSIC TO THE STATE-SPACE LEARNING MODEL

Because the force-field was kept constant on all trials in which it was applied but the actual force experienced varied depending on the movement velocity, we followed Thoroughman and Shadmehr (2000) in representing the force input to the model, $f(n)$, as a discrete scalar representing force-field magnitude and assigning it values of 1,0 , or -1 corresponding to field A, catch, or field B trials, respectively. Thus the compliance computed from this model has units of angular error and it corresponds to the aiming error directly induced by the force field. Although this measure of compliance is nonstandard, it is intrinsically related to the usual measure of compliance. The relationship between these measures is derived in the following text.

Here we compute the relationship between force-field induced angular displacement and real arm compliance. Compliance is the constant of proportionality between force and displacement. Because the force-field induced displacement at a certain point in time is caused by forces experienced up to that time, it is reasonable to define the effective compliance at a certain time point, $D_{1}$, as the constant of proportionality between the average force experienced up to that time point and the displacement at that time point

$$
X(t)=D_{1} \cdot F x_{\mathrm{AVG}(0 \rightarrow t)}
$$

Note that in the force-field used in these experiments, $x$-direction force depends on $y$-direction velocity, and the force-field viscosity, $C$. That is, $F x_{\mathrm{AVG}(0 \rightarrow t)}=C \cdot V y_{\mathrm{AVG}(0 \rightarrow t)}$. Average velocity is simply the ratio between change in position and change in time

$$
V y_{\mathrm{AVG}(0 \rightarrow t)}=\frac{Y(t)}{t}
$$

Combining these three equations yields

$$
X(t)=\frac{D_{1} \cdot \mathrm{C} \cdot Y(t)}{t}
$$

if we consider the time point $300 \mathrm{~ms}$ after movement onset, we get

$$
\begin{aligned}
& X_{300}=\frac{D_{1} \cdot C \cdot Y_{300}}{t} \\
& \frac{X_{300}}{Y_{300}}=\frac{C}{300 \mathrm{~ms}} \cdot D_{1}
\end{aligned}
$$

Note that our model's compliance measure, angular displacement, is closely related to the ratio of $x$ and $y$ displacements, and therefore is closely related to stiffness

$$
\phi_{300}=\arctan \left(\frac{X_{300}}{Y_{300}}\right) \approx \frac{X_{300}}{Y_{300}}=\frac{C}{300 \mathrm{~ms}} \cdot D_{1}
$$

For angular displacements of $<15^{\circ},\left(\frac{X_{300}}{Y_{300}}\right)$ is within $2.2 \%$ of $\frac{X_{300}}{Y_{300}}$, and for angular displacements of $<20^{\circ},\left(\frac{X_{300}}{Y_{300}}\right)$ is within $3.8 \%$ of $\frac{X_{300}}{Y_{300}}$, yielding a nearly linear relationship between $\phi_{300}$ and $D_{1}$ for the range of angular displacements in our data. Thus $\phi_{300}$ can be approximately converted to $D_{1}$ by simply multiplying it by $\frac{300 \mathrm{~ms}}{C}$. 


\section{G R A N T S}

This work was supported by National Institute of Neurological Disorders and Stroke Grants NS-37422 and NS-16375 and a grant from the Hereditary Disease Foundation.

\section{REFERENCES}

Atkeson CG. Learning arm kinematics and dynamics. Ann Rev Neurosci 12: 157-183, 1989.

Aylward EH, Anderson NB, Bylsma FW, Wagster MV, Barta PE, Sherr M, Feeney J, Davis A, Rosenblatt A, Pearlson GD, and Ross CA. Frontal lobe volume in patients with Huntington's disease. Neurology 50: 252-258, 1998.

Aylward EH, Brandt J, Codori AM, Mangus RS, Barta PE, and Harris GJ. Reduced basal ganglia volume associated with the gene for Huntington's disease in asymptomatic at-risk persons. Neurology 44: 823-828, 1994.

Bachevalier, J. Ontogenetic development of habit and memory formation in primates. Ann NY Acad Sci 608: 457-484, 1990.

Bastian AJ, Martin TA, Keating JG, and Thach WT. Cerebellar Ataxia: abnormal control of interaction torques across multiple joints. $J$ Neurophysiol 76: 492-509, 1996.

Bastian AJ, Zackowski KM, and Thach WT. Cerebellar ataxia: torque deficiency or torque mismatch between joints? J Neurophysiol 83: 30193030, 2000.

Bhushan $\mathbf{N}$ and Shadmehr R. Computational architecture of human adaptive control during learning of reaching movements in force fields. Biol Cybern 81: 39-60, 1999.

Chen L, Bao S, Lockard JM, Kim JK, and Thompson RF. Impaired classical eyeblink conditioning in cerebellar-lesioned and Purkinje cell degeneration (pcd) mutant mice. J Neurosci 16: 2829-2838, 1996.

Conditt MA, Gandolfo F, and Mussa-Ivaldi FA. The motor system does not learn the dynamics of the arm by rote memorization of past experience. J Neurophysiol 78: 554-560, 1997.

Cordo PJ. Kinesthetic control of a multijoint movement sequence. J Neurophysiol 63: 161-172, 1990.

Criscimagna-Hemminger SE, Donchin O, Gazzaniga MS, and Shadmehr R. Learned dynamics of reaching movements generalize from dominant to nondominant arm. J Neurophysiol 89: 168-176, 2003.

Desmurget M, Gaveau V, Vindras P, Turner RS, Broussolle E, and Thobois S. On-line motor control in patients with Parkinson's disease. Brain 127: 1755-1773, 2004.

Donchin O, Francis JT, and Shadmehr R. Quantifying generalization from trial-by-trial behavior of adaptive systems that learn with basis functions: theory and experiments in human motor control. J Neurosci 23: 9032-9045, 2003.

Dugas C and Smith AM. Responses of cerebellar Purkinje cells to slip of a hand-held object. J Neurophysiol 67: 483-495, 1992.

Fellows S, Schwarz M, Schaffrath C, Domges F, and Noth J. Disturbances of precision grip in Huntington's disease. Neurosci Lett 226: 103-106, 1997.

Fernandez-Ruiz J, Diaz R, Hall-Haro C, Vergara P, Mischner J, Nunez L, Drucker-Colin R, Ochoa A, and Alonso ME. Normal prism adaptation but reduced after-effect in basal ganglia disorders using a throwing task. Eur J Neurosci 18: 689-694, 2003.

Folstein SE, Jensen B, Leigh RJ, and Folstein MF. The measurement of abnormal movement: methods developed for Huntington's disease. Neurobehav Toxicol Teratol 5: 605-609, 1983.

Goodkin HP, Keating JG, Martin TA, and Thach WT. Preserved simple and impaired compound movement after infarction in the territory of the superior cerebellar artery. Can J Neurol Sci 20, Suppl. 3: S93-S104, 1993.

Gusella JF, Wexler NS, Conneally PM, Naylor SL, Anderson MA, Tanzi RE, Watkins PC, Ottina K, Wallace MR, and Sakaguchi AY. A polymorphic DNA marker genetically linked to Huntington's disease. Nature 306: $234-238,1983$.

Harris GJ, Aylward EH, Peyser CE, Pearlson GD, Brandt J, RobertsTwillie JV, Barta PE, and Folstein SE. Single photon emission computed tomographic blood flow and magnetic resonance volume imaging of basal ganglia in Huntington's disease. Arch Neurol Arch Neurol : 316-324, 1996.

Hefter H, Homberg V, Lange HW, and Freund HJ. Impairment of rapid movement in Huntington's disease. Brain 110: 585-612, 1987.

Hoff B and Arbib MA. Models of trajectory formation and temporal interaction of reach and grasp. J Mot Behav 25: 175-192, 1993.
Holm S. A simple sequentially rejective multiple test procedure. Scan J Statist 6: 65-70, 1979.

Ijspeert AJ, Nakanishi J, and Schaal S. Movement imitation with nonlinear dynamical systems in humanoid robots. Proc IEEE Intern Conf Robotics Automat: 1398-1403, 2002.

Jordan MI and Rumelhart DE. Forward models: supervised learning with a distal teacher. Cognit Sci 16: 307-354, 1992.

Kawato M. Adaptation and learning in control of voluntary movement by the central nervous system. Adv Robotics 3: 229-249, 1989.

Kawato $\mathbf{M}$ and Gomi H. A computational model of four regions of the cerebellum based on feedback-error learning. Biol Cybern 68: 95-103, 1992.

Kirkwood SC, Siemers E, Bond, C.a.C, Christian JC, and Foroud T. Confirmation of subtle motor changes among presymptomatic carriers of the Huntington Disease Gene. Arch Neurol 57: 1040-1044, 2000.

Marsden CD, Merton PA, Morton HB, Adam JER, and Hallett M. Automatic and volunary responses to muscle stretch in man. Prog Clin Neurophysiol 4: 167-177, 1978.

Martin TA, Keating JG, Goodkin HP, Bastian AJ, and Thach WT. Throwing while looking through prisms. I. Focal olivocerebellar lesions impair adaptation. Brain 119: 1183-1198, 1996.

Maschke M, Gomez CM, Ebner TJ, and Konczak J. Hereditary cerebellar ataxia progressively impairs force adaptation during goal-directed arm movements. J Neurophysiol 91: 230-238, 2004.

Meyer BU, Noth J, Lange HW, Bischoff C, Machetanz J, Weindl A, Roricht S, Benecke R, and Conrad B. Motor responses evoked by magnetic brain stimulation in Huntington's disease. EEG Clin Neurophysiol 85: 197-208, 1992.

Miall RC, Weir DJ, and Stein JF. Manual tracking of visual targets by trained monkeys. Behav Brain Res 20: 185-201, 1986.

Miall RC and Wolpert DM. Forward models for physiological motor control. Neural Networks 9: 1265-1279, 1996.

Mishkin M and Appenzeller T. The anatomy of memory. Sci Am 256: 80-89, 1987.

Mishkin M, Malamut B, and Bachevalier J. Memories and habits: two neural systems. In: Neurobiology of Learning and Memory, Lynch G.a.M. London: Guilford Press, 1984, 65-77.

Mussa-Ivaldi FA, Hogan N, and Bizzi E. Neural, mechanical, and geometric factors subserving arm posture in humans. J Neurosci 1985 10: 2732-2743, 1985.

Nezafat R, Shadmehr R, and Holcomb HH. Long-term adaptation to dynamics of reaching movements: a PET study. Exp Brain Res 140: 66-76, 2001.

Noth J, Podoll K, and Friedemann HH. Long-loop reflexes in small hand muscles studied in normal subjects and in patients with Huntington's disease. Brain 108: 65-80, 1985.

Paulsen JS, Butters N, Salmon DP, and Heindel WC. Prism adaptation in Alzheimer's and Huntington's disease. Neuropsychology 7: 73-81, 1993.

Penney JB, Jr, Young AB, Shoulson I, Starosta-Rubenstein S, Snodgrass SR, Sanchez-Ramos J, Ramos-Arroyo M, Gomez F, Penchaszadeh G, Alvir, J, and. Huntington's disease in Venezuela: 7 years of follow-up on symptomatic and asymptomatic individuals. Mov Disord 5: 93-99, 1990.

Petersen N, Christensen LOD, Morita H, Sinkaer T, and Nielsen J. Evidence that a transcortical pathway contributes to stretch reflexes in the tibialis anterior muscle in man. J Physiol 512: 267-276, 1998.

Robertson EM and Miall RC. Visuomotor adaptation during inactivation of the dentate nucleus. Neuroreport 10: 1029-1034, 1999.

Rothwell JC. Long latency reflexes of human arm muscles in health and disease. In: New Trends and Advanced Techniques in Clinical Neurophysiology, edited by Rossini PM. Amsterdam: Elsevier, 1990, 251-263.

Sanes JN, Dimitrov B, and Hallett M. Motor learning in patients with cerebellar dysfunction. Brain 113: 103-120, 1990.

Scheidt RA, Dingwell JB, and Mussa-Ivaldi FA. Learning to move amid uncertainty. J Neurophysiol 86: 971-985, 2001.

Schwarz M, Fellows SJ, Schaffrath C, and Noth J. Deficits in sensorimotor control during precise hand movements in Huntington's disease. Clin Neurophysiol 112: 95-106, 2001.

Shadmehr R and Brashers-Krug T. Functional stages in the formation of human long-term motor memory. J Neurosci 17: 409-419, 1997.

Shadmehr R and Mussa-Ivaldi FA. Adaptive representation of dynamics during learning of a motor task. J Neurosci 14: 3208-3224, 1994.

Shadmehr R and Wise SP. Motor learning and memory for visually guided reaching. In: The Cognitive Neurosciences III, edited by Gazzaniga MS. Cambridge, MA: MIT Press, 2004, p. 353-375. 
Shadmehr R and Wise SP. The Computational Neurobiology of Reaching and Pointing: A Foundation for Motor Learning. Cambridge, MA: MIT Press, 2005.

Siemers E, Foroud T, Bill DJ, Sorbel J, Norton JA, Hodes ME, Niebler G, Conneally PM, and Christian JC. Motor changes in presymptomatic Huntington disease gene carriers. Arch Neurol 53: 487-492, 1996.

Smith MA, Brandt J, and Shadmehr R. The motor dysfunction in Huntington's Disease begins as a disorder in error feedback control. Nature 403: $544-549,2000$

Thilmann AF, Schwarz M, Topper R, Fellows SJ, and Noth J. Different mechanisms underlie the long-latency stretch reflex response of active human muscle at different joints. J Physiol 444: 631-643, 1991.

Thompson PD, Berardelli A, Rothwell JC, Day BL, Dick JP, Benecke R, and Marsden CD. The coexistence of bradykinesia and chorea in Huntington's disease and its implications for theories of basal ganglia control of movement. Brain 111: 223-244, 1988.

Thoroughman KA and Shadmehr R. Learning of action through adaptive combination of motor primitives. Nature 407: 742-747, 2000.

Todorov E and Jordan MI. Optimal feedback control as a theory of motor coordination. Nat Neurosci 5: 1226-1235, 2002.
Topka H, Konczak J, Schneider K, Boose A, and Dichgans J. Multijoint arm movements in cerebellar ataxia: abnormal control of movement dynamics. Exp Brain Res 119: 493-503, 1998.

Topka H, Valls-Sole J, Massaquoi SG, and Hallett M. Deficit in classical conditioning in patients with cerebellar degeneration. Brain 116: 961-969, 1993.

Topper R, Schwarz M, Podoll K, Domges F, and Noth J. Absence of frontal somatosensory evoked potentials in Huntington's disease. Brain 116: 87$101,1993$.

Willingham DB and Koroshetz WJ. Evidence for dissociable motor skills in Huntington's disease patients. Psychobiology 21: 173-182, 1993.

Willingham DB, Koroshetz WJ, and Peterson EW. Motor skills have diverse neural bases: spared and impaired skill acquisition in Huntington's disease. Neuropsychology 10: 315-321, 1996.

Wolpert DM, Ghahramani Z, and Jordan MI. An internal model for sensorimotor integration. Science 269: 1880-1882, 1995.

Wolpert DM, Goodbody SJ, and Husain M. Maintaining internal representations: the role of the human superior parietal lobe. Nat Neurosci 1: 529-533, 1998 